\title{
Lyn regulates epithelial-mesenchymal transition in CS-exposed model through Smad2/3 signaling
}

Xiaobo Liang ${ }^{1,2+}$, Xiang $\mathrm{He}^{3,4+}$, Yin $\mathrm{Li}^{5}$, Junyi Wang ${ }^{3,4}$, Dehong Wu${ }^{3,4}$, Xiefang Yuan ${ }^{1}$, Xiaoyun Wang ${ }^{1}$ and Guoping Li, $i^{1,3,4^{*}}$

\begin{abstract}
Background: Chronic obstructive pulmonary disease (COPD) is characterized by airflow limitation that is progressive and not fully reversible. Cigarette smoking is one of the most commonly and important risk factors for COPD, which contributes to airway remodeling, the outstanding pathological changes in COPD. One potential mechanism which might be important for airway remodeling is the process called epithelial-mesenchymal transition (EMT). However, the underlying molecular mechanisms of EMT in CS-induced COPD are still poorly understood.
\end{abstract}

Methods: Two Gene Expression Omnibus (GEO) datasets (GSE108134 and GSE5058) were combined to identify the key genes involved in COPD. Then, single-gene analysis of Lyn was performed. Lyn expression was confirmed in patients with COPD. 16HBE cells were treated with cigarette smoking extracts (CSE). Wild type (WT) C57BL/6 J mice and Lyn+/+ transgenic mice were exposed to CSE to establish CS-exposed model. Pathological changes were observed by hematoxylin-eosin staining. The expression levels of EMT markers were examined by using western blot and immunofluorescence. The expression and phosphorylation levels of Lyn and Smad2/3 were detected as well.

Results: The gain of mesenchymal markers vimentin and a-SMA with a concomitant loss of E-cadherin was observed in both in vivo and in vitro studies. Meanwhile, cigarette smoking extracts (CSE) induced EMT in 16HBE cells in a timeand dose- dependent manner. Furthermore, by analyzing GEO datasets and using molecular methods, we explored a kinase, Lyn, its expression correlated with the expression of E-cadherin, vimentin and a-SMA in CS-exposed model. Moreover, we found that EMT induced by CSE was regulated by activated Lyn through phosphorylation of Smad2/3.

Conclusions: In summary, we found that Lyn regulates epithelial-mesenchymal transition in CS-exposed model through Smad2/3 signaling. As a kinase, Lyn is "druggable", and might provide a therapeutic opportunity for targeting EMT. Therefore, our research might provide a new method to treat COPD by targeting Lyn kinase specifically.

Keywords: Lyn, EMT, COPD, Smad2/3

\footnotetext{
*Correspondence: Izlgp@163.com

${ }^{+}$Xiaobo Liang and Xiang He contributed equally to this work.

${ }^{1}$ Inflammation \& Allergic Diseases Research Unit, Affiliated Hospital of

Southwest Medical University, Luzhou 646000, China

${ }^{3}$ Laboratory of Allergy and Inflammation of Allergy Department, Chengdu

Institute of Respiratory Health, the Third People's Hospital of Chengdu,

Affiliated Hospital of Southwest Jiaotong University, Chengdu 610031, China

Full list of author information is available at the end of the article
}

(c) The Author(s). 2019 Open Access This article is distributed under the terms of the Creative Commons Attribution 4.0 International License (http://creativecommons.org/licenses/by/4.0/), which permits unrestricted use, distribution, and reproduction in any medium, provided you give appropriate credit to the original author(s) and the source, provide a link to the Creative Commons license, and indicate if changes were made. The Creative Commons Public Domain Dedication waiver (http://creativecommons.org/publicdomain/zero/1.0/) applies to the data made available in this article, unless otherwise stated. 


\section{Background}

Chronic obstructive pulmonary disease (COPD), which is characterized by continuous limitation of airflow, is a chronic inflammatory pulmonary disease. According to the data of World Health Organization, COPD will be the third leading cause of death all over the world by the year 2030 [1]. Cigarette smoking is one of the most commonly and important risk factors for COPD. Although a few of individuals with COPD had never smoked, personal exposure to tobacco smoke like cigarette smoking is one of the best known risk factor for COPD [2]. It was found that the prevalence rate of COPD in smokers is 3 to 5-times higher than that in nonsmokers [3]. However, the underlying molecular mechanisms of cigarette smoking in COPD development are still poorly understood.

Remodeling of the airways is one of the outstanding pathophysiological changes in COPD, which consists of detrimental changes in structural tissues and cells including epithelial metaplasia, smooth muscle hyperplasia, goblet cell hypertrophy, and airway wall thickening $[4,5]$. Airway remodeling largely leads to airway obstruction and causes the airflow limitation seen in patients with COPD. However, the mechanism of airway remodeling is not fully elucidated. One potential mechanism which might be important for this pathology is the process of epithelial-mesenchymal transition (EMT) $[6,7]$. EMT is a biological process in which epithelial cells lose their abilities of cell-cell adhesion and polarity with rearrangement of the cytoskeleton, and the cells acquire the abilities of producing ECM components, invasion and migration $[8,9]$. Biomarkers of epithelial cells like E-cadherin and ZO-1 are down-regulated during EMT, whereas mesenchymal markers, VIM (Vimentin) and $\alpha$-SMA (alpha-smooth muscle actin), are up-regulated $[10,11]$. Although the mechanism of EMT in COPD has not been fully illustrated, Smad2/3 signaling is believed to be involved in EMT. Smad2 and Smad3 belong to Smad-transcription factors, which are activated by TGF $\beta$ receptors. Phospho-activated $\operatorname{Smad} 2 / 3$ form a complex with Smad4, then the complex translocates to the nucleus. In the nucleus, this Smad complex binds to promoter regions of target genes and regulates expression of numerous target genes [12-14]. Recent studies also suggest that Smad2 and/or Smad3 are associated with EMT-related cancer models [15-17].

Lyn is a Src-family kinase, a family of non-receptor tyrosine kinases, which plays vital roles in signal regulation and transduction. Lyn has been implicated in regulation of cellular events, such as survival, proliferation and apoptosis depending on cell types and stimulus [18]. Studies have shown that Lyn plays a crucial role in Kaposi's Sarcoma, autoimmune disease and hepatic cirrhosis as well as allergic bronchial asthma [19, 20]. In addition, Lyn is associated with metastasis and may promote EMT in tumor progression [21]. In this study, we explored the underlying mechanism of how Lyn regulates EMT in CS-exposed model. Lyn was activated in CS-exposed model and promoted EMT both in vitro and in vivo studies. Furthermore, the phosphorylation of Smad2/3 was up-regulated by activated Lyn. Our results suggest that Lyn is the activator of Smad2/3, the major transcription factors involved in EMT. Therefore, specific targeting of Lyn might be an effective therapeutic approach to treat COPD by inhibition of EMT.

\section{Materials and methods \\ Reagents}

Anti-Lyn(Santa Cruz, CA, sc-15), anti-Vimentin (Santa Cruz,sc-6260), anti-Smad2/3(Santa Cruz, CA, sc-8332), anti-p-Smad2/3(Santa Cruz, CA, sc-11,769), and anti- $\beta$ actin(Santa Cruz, CA, sc-130,656) were purchased from Santa Cruz Biotechnology. Anti-E-cadherin (Abcam, ab11512), and anti- $\alpha$-SMA (Abcom,ab5694) were purchased from Abcam Biotechnology. Anti-p-Lyn (CST, \#2731) was purchased from Cell Signaling Technology. Nonsilencing siRNA control, Lyn-specific siRNA were purchased from Santa Cruz Biotechnology (Santa Cruz, CA). Recombinant lentivirus containing Lyn or a GFPonly virus control was obtained from Cyagen (Shanghai, China).

\section{Animals and cell line}

The construction of the $\mathrm{Lyn}^{+/+}$transgenic mice with inbred C57BL/6 J genomic background has been previously described [22]. Genotyping was confirmed by western blotting. Wild type (WT) C57BL/6 J mice at 8 to 10 weeks of age were obtained from Tengxin Biotechnology Co., Ltd. (Chongqing, China). $\mathrm{Lyn}^{++}$TG mice and WT mice were maintained in a specific pathogenfree facility and routinely monitored. All animal experiments were performed and approved in accordance with the guidelines of the Institutional Animal Care and Use Committee in Southwest Medical University. The 16HBE cell line was obtained from Wang [22].

\section{Bioinformatics and computational analysis}

The published microarray datasets, GSE108134 and GSE5058, were obtained from public database NCBI. Airway epithelial cells from COPD-smoker or Nonsmoker were obtained by bronchoscopy and brushing in both GEO datasets. The sample numbers of Non-smoker group are 39, COPD-smoker group are 69. The matrix data of GSE108134 and GSE5058 were merged and normalized by sva package with $R$ software. Gene set enrichment analysis was performed with GESA 3.0 according to previous publications [23, 24]. 


\section{Establishment of murine CS-exposed model}

To establish a murine CS-exposed model, 6 to10-weeks old $\mathrm{Lyn}^{+/+}$transgenic mice and WT mice were treated with CSE combined with lipopolysaccharide (LPS), 5 mice in each group were used $[25,26]$. The mice were treated with CSE for 5 days per week for a total of 6 weeks, $10 \mu \mathrm{g}$ LPS was given twice a week for 6 weeks. Control mice were given PBS alone.

\section{Histology}

Lung tissues were fixed in 10\% neutral-buffered formalin for $24 \mathrm{~h}$ and then embedded in paraffin. The sections $(5 \mu \mathrm{m})$ of the lung specimens were stained with standard hematoxylin-eosin staining (H\&E) methods to assess histology. Histological review was performed in a blinded fashion by two independent pulmonary observers.

\section{Cell culture, viral infection and transfection}

Human Lyn cDNA was amplified and cloned into the pLV.ExBi.P/Puro-EF1 $\alpha$-IRES-eGFP/ pLV.Des3d.P/Puro vector. The vector expressing Lyn was constructed and transfected into human embryo kidney cells $293 \mathrm{~T}$ cells to create infectious lentiviral vector [22]. Human airway epithelial cells 16HBE were obtained from American Type Culture Collection (ATCC; Manassas, USA). $16 \mathrm{HBE}$ cells were maintained in Dulbecco's modified Eagle's medium (DMEM) with $10 \%$ fetal bovine serum at $37^{\circ} \mathrm{C}$ in with $5 \% \mathrm{CO} 2.16 \mathrm{HBE}$ cells were grown to $70 \%$ confluence in DMEM culture media containing $10 \%$ fetal bovine serum. 16HBE cells were transfected with recombinant lentivirus containing Lyn or a GFP-only virus control according to the manufacturer's instructions. $16 \mathrm{HBE}$ cells were transfected with lentivirus at a multiplicity of infection (MOI) of 5-10. The supernatant was exchanged for fresh medium after $12 \mathrm{~h}$-inoculation. For Lyn interfering RNA (siRNA), cells were transfected with 20uM small interfering RNA using Lipofectamine 2000 according to the manufacturer's instructions. The media were changed after $6 \mathrm{~h}$-transfection. Cigarette smoke extracts (CSE) were prepared as previously outlined [27].The cells were treated with 0\% CSE, 2.5\% CSE and 5\% CSE for $72 \mathrm{~h}$, and 5\% CSE at $6 \mathrm{~h}$, $12 \mathrm{~h}, 24 \mathrm{~h}, 48 \mathrm{~h}, 72 \mathrm{~h}$.

\section{Immunofluorescence}

Frozen lung tissues sections $(5 \mu \mathrm{m})$ and cells were fixed with ice-cold methanol and permeabilized in PBS containing $0.25 \%$ Triton $\mathrm{X}-100$ for $10 \mathrm{~min}$ at room temperature. Nonspecific binding was blocked for $1 \mathrm{~h}$ with 1\% BSA (Sigma-Aldrich, B2064) in PBS containing $0.05 \%$ Tween 20. Specimens were then incubated with antibodies against E-cadherin, vimentin and $\alpha$-SMA. FITC-conjugated or TRITC-conjugated secondary Abs were used to probe the primary antibodies. After the specimens were washed, nuclei were stained with 4'-6diamidino-2-phenylindole dihydrochloride (DAPI, Invitrogen). The specimens were analyzed using an SP5 Leica confocal microscope with Leica Application Suite Software (Version number: 14.0.0162, Leica, German). Isotype control was replaced the Abs as the negative control.

\section{Western blotting}

Lung tissues or cells were homogenized in liquid nitrogen and re-suspended in radioimmunoprecipitation assay (RIPA) buffer with optional protease inhibitor cocktail (Roche Applied Science, Indianapolis, USA). The protein concentrations were measured using a Nanodrop Instrument (Epoch, BioTek). Protein samples were separated in $8-10 \%$ SDS-PAGE at $100 \mathrm{~V}$ for $90 \mathrm{~min}$ and transferred onto a microporous polyvinylidene difluoride (PVDF) membrane with $250 \mathrm{~mA}$ current for 1 $\mathrm{h}$ using a wet transfer method. The PVDF membranes were incubated with antibodies against E-cadherin, vimentin, $\alpha$-SMA, Smad2/3, p-Smad2/3, $\beta$-actin, Lyn, and p-Lyn. Horseradish peroxidase (HRP)-conjugated secondary antibodies were used to bind corresponding primary antibodies and visualized via an enhanced chemiluminescence kit. The band intensity was evaluated against the loading control to derive a ratio value using Quantity One software.

\section{Statistical analysis}

All statistical analyses were performed using the SPSS 17.0 software (Chicago, IL, USA). Graphs were made in Graphpad Prism 7 (GraphPad Software Inc., LaJolla, CA). Quantitative data are presented as the mean \pm s.d. Multiple group comparisons were analyzed using a oneway ANOVA. Two group comparisons were analyzed by using the Tukey-Kramer post-test or Dunnett's T3. Statistical significance was defined as a $P$ value $<0.05$.

\section{Results}

The expression levels of EMT makers correlate with Lyn in COPD-smoker patients

We combined two GEO datasets (GSE108134 and GSE5058) to identify the key genes involved in COPD. Gene Set Enrichment Analysis (GSEA) showed that Lyn kinase expressed differentially between Non-smoker group $(n=39)$ and COPD-smoker group $(n=69)$ (Fig. 1a). Notably, single-gene analysis of Lyn revealed that high expression of Lyn was associated with adherens junction (Fig. 1b). To investigate the correlation between Lyn and EMT in COPD-smoker patients, we analyzed the expression levels of Lyn, vimentin and $\alpha$ SMA. We found that the expression level of Lyn was significantly up-regulated in COPD-smoker patients compared with Non-smoker ( $p=0.007$, Fig. 1c). Meanwhile, 


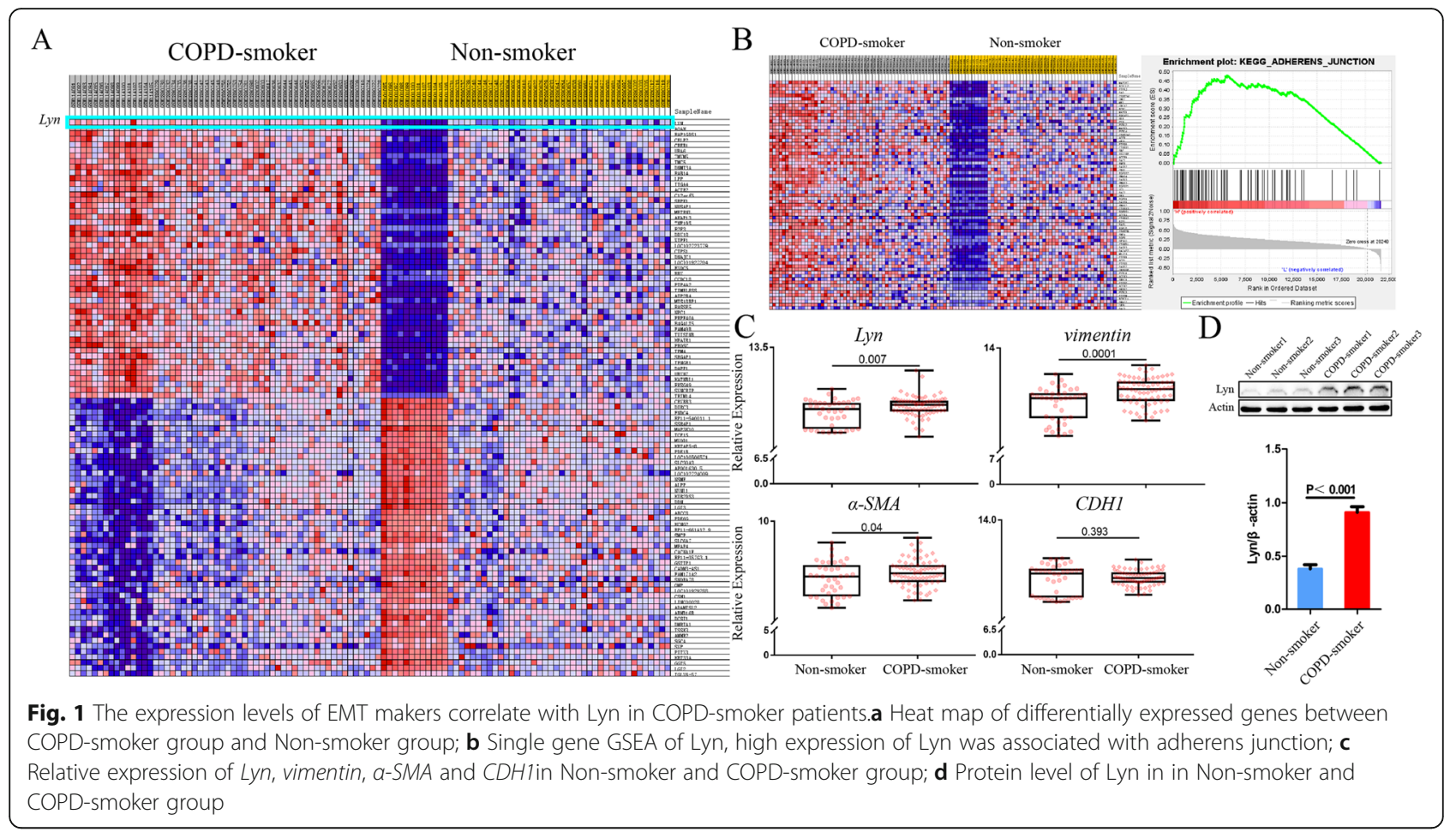

the expression levels of vimentin $(p=0.0001)$ and $\alpha$ SMA $(p=0.04)$, two molecular markers of EMT, were increased as well. However, there was no difference in the expression of E-cadherin $(P=0.393)$. To validate the microarray data, western blot was applied. It also showed that the protein level of Lyn was significantly increased in COPD-smoker patients (Fig. 1d).

\section{Cigarette smoke extracts (CSE) induces EMT in a time- and dose-dependent manner}

Cigarette smoking is one of the most commonly identifiable risk factors for COPD. In order to investigate whether CSE induces EMT in vitro, different time-points or concentrations of CSE were performed in 16HBE cells and expression levels of EMT markers were assessed by using western blot analysis. The data showed that CSE down-regulated E-cadherin protein level and up-regulated vimentin and $\alpha$-SMA protein levels in a time- and dose-dependent manner (Fig. 2).

\section{EMT induced by CSE is regulated by activated Lyn}

To further explore the role of Lyn in EMT that induced by CSE, Lyn was over-expressed in 16HBE cells and the expression levels of EMT makers were examined by western blot. Cells were treated with 5\% CSE for $72 \mathrm{~h}$. Compared with those in mock group, the protein levels of vimentin and $\alpha$-SMA in mock/CSE and $\mathrm{Lyn}^{+/+} / \mathrm{CSE}$ were increased (Fig. 3a, c and d). In the meantime, Ecadherin protein levels were decreased in mock/CSE and
Lyn $^{+/+} /$CSE compared with those in mock treatment (Fig. 3a, b). However, no changes in the protein levels of the three EMT markers were observed between $\mathrm{Lyn}^{+/+}$ and mock group (Fig. 3). Therefore, it forced us to detect the phosphorylation levels of Lyn in different groups. It showed that although the protein level of Lyn was up-regulated in $\mathrm{Lyn}^{+/+}$cells, the phosphorylation level of Lyn in this group was not changed (Fig. 3a, e). Whereas the phosphorylation levels of Lyn were increased in mock/CSE and $\mathrm{Lyn}^{+/+} / \mathrm{CSE}$ compared with non-treated groups including mock and $\mathrm{Lyn}^{+/+}$cells (Fig. 3a, e). These results revealed that both the expression and phosphorylation of Lyn were induced by CSE, and the phosphorylation level of Lyn may play a key role in regulation of EMT. Additionally, we found that the phosphorylation levels of Smad2/3 were increased in mock/CSE and $\mathrm{Lyn}^{+/+} / \mathrm{CSE}$, but there was no difference in $\mathrm{Lyn}^{+/+}$cells (Fig. 3a, f).

\section{Lyn regulates CSE-induced EMT by phosphorylation of Smad2/3}

Lyn is a kinase, which functions primarily by phosphorylation of downstream effectors. To investigate whether the kinase activity of Lyn is required for the regulation of EMT, the expression of Lyn was inhibited by using siRNA. The EMT markers expression levels were checked by immunofluorescence (Fig. 4a, b). Compared with that in mock/CSE, the fluorescence intensity of Ecadherin in si-Lyn/CSE was increased $(p=0.004)$, 
A

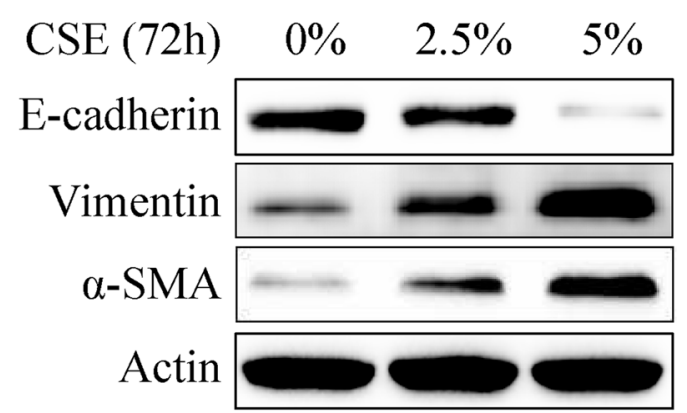

B

C

D
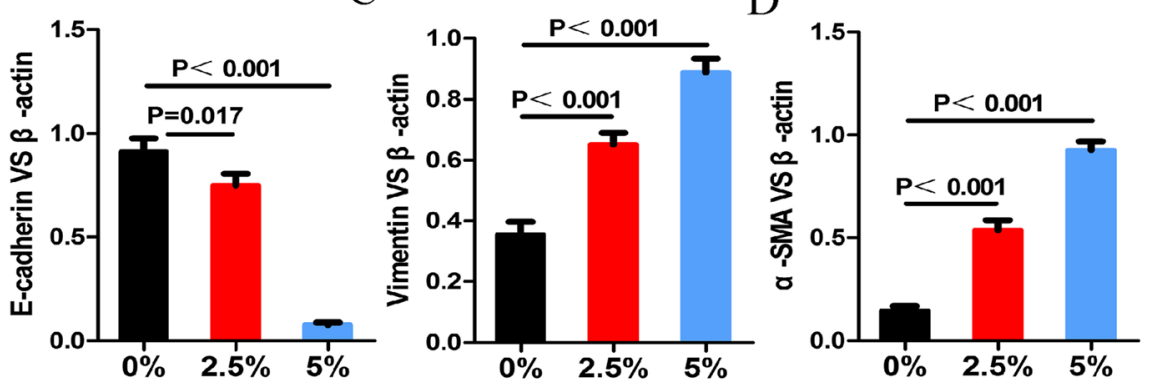

$\mathrm{E}$
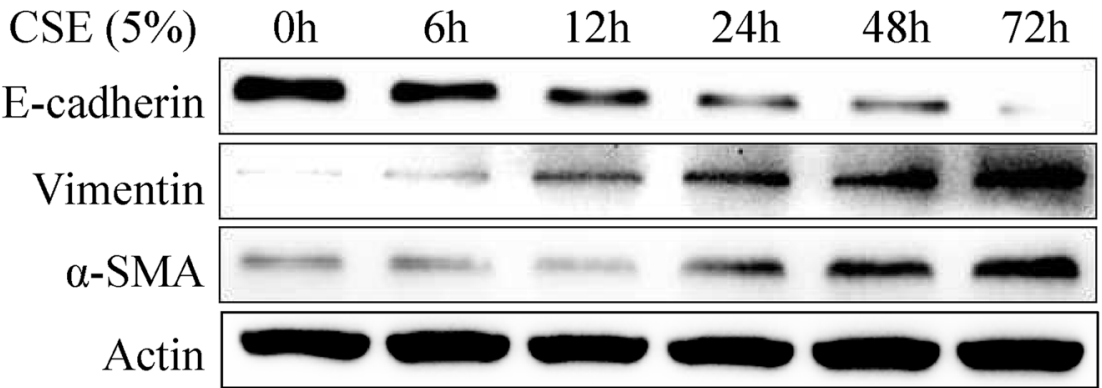

$\mathrm{F}$

G

$\mathrm{H}$
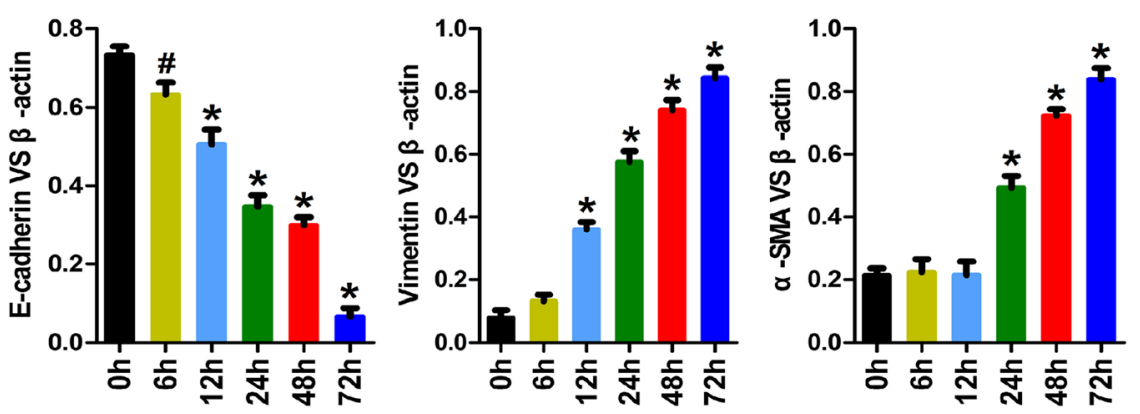

Fig. 2 CSE induces EMT in a time- and dose-dependent manner. a 16HBE cells were treated with 0, 2.5 and 5\% CSE, respectively. Then the expression of EMT markers were detected by western blot; b, c and $\mathbf{d}$ Statistic data of each EMT markers in Western blot analysis; e 16 HBE cells were treated with 5\% CSE at different time points; $\mathbf{f}, \mathbf{g}$ and $\mathbf{h}$ Statistic data of each EMT markers in Western blot analysis. The data represent means \pm s.d. All data are representative of three experiments

whereas the fluorescence intensity of vimentin in si-Lyn/ CSE was decreased $(p=0.042)$. Similar results were obtained in western blot analysis (Fig. 4c). The protein level of $\alpha$-SMA was decreased in si-Lyn /CSE compared with mock/CSE as well (Fig. 4c, f). Furthermore, the phosphorylation level of Lyn in si-Lyn /CSE was significantly down-regulated compared with mock/CSE $(p<0.001$, Fig. $4 \mathrm{c}, \mathrm{h})$. Moreover, the phosphorylation level of Smad2/3, which was increased by CSE, was decreased after the expression and phosphorylation of 
A

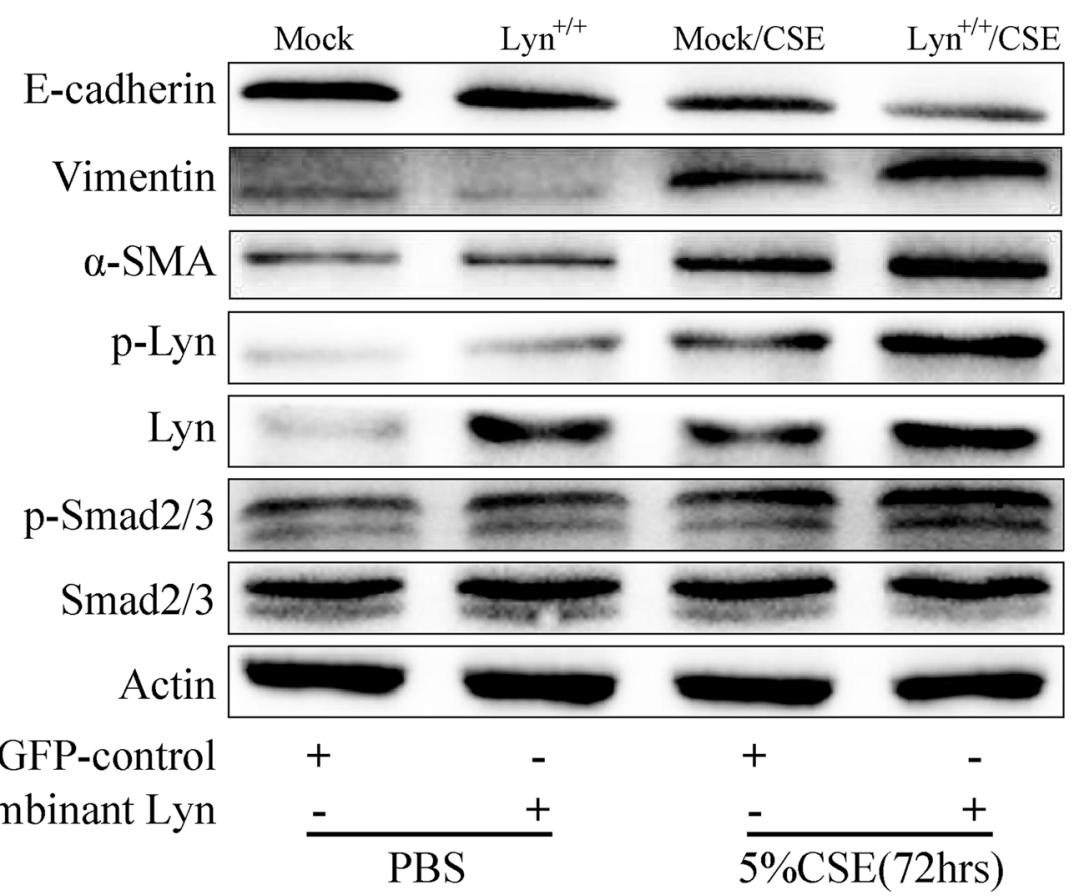

B

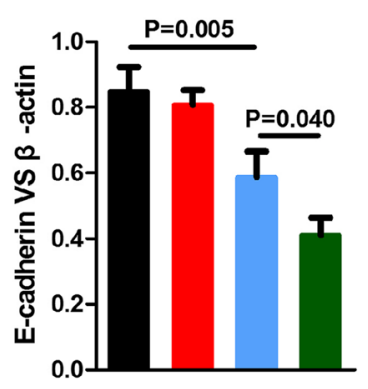

E
C

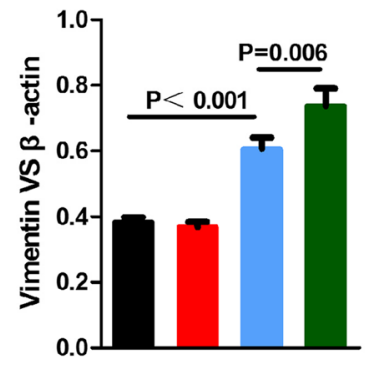

F
D

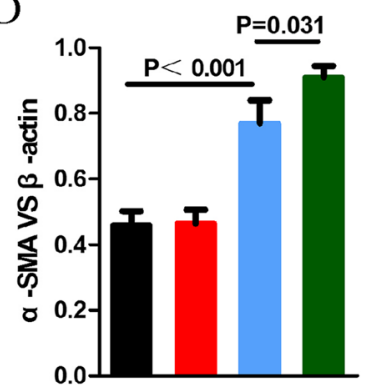

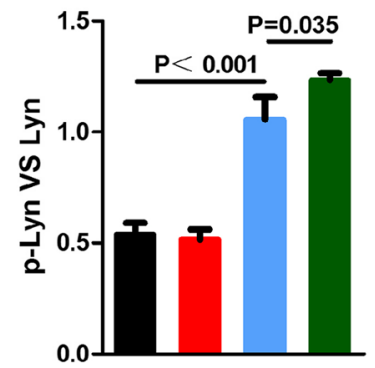
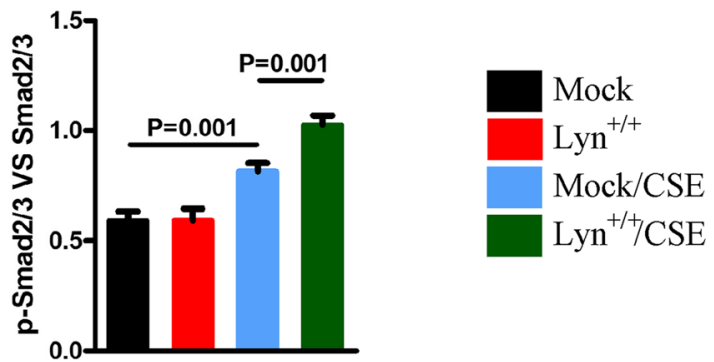

Fig. 3 Lyn kinase activity regulates EMT. a The expression of EMT markers, Lyn, Smad2/3, and phosphorylation of Lyn and Smad2/3 in 16HBE cells treated with $5 \%$ CSE for $72 \mathrm{~h}$ were detected by western blot; $\mathbf{b} \mathbf{c}, \mathbf{d}$, e, and $\mathbf{f}$ Statistic data of each indicated genes in Western blot analysis. Mock, $16 \mathrm{HBE}$ cells were infected with GFP-only virus control. Lyn ${ }^{+/+}, 16 \mathrm{HBE}$ cells were infected with recombinant lentivirus containing Lyn. Mock/CSE, $16 \mathrm{HBE}$ cells were treated with $5 \%$ CSE after infected with GFP-only virus control. Lyn ${ }^{+/+} / \mathrm{CSE}, 16 \mathrm{HBE}$ cells were treated with $5 \%$ CSE after infected with recombinant lentivirus containing Lyn. The data represent means \pm s.d. All data are representative of three experiments

Lyn were inhibited ( $p=0.015$, Fig. $4 \mathrm{c}, \mathrm{h})$. Recent studies discovered that phospho-activated Smad2/3 functions as major transcription factor to mediate EMT $[14,28]$. Taken together, these data support that Lyn expression and activity regulate EMT by phosphorylation of Smad2/3.
Effect of Lyn on EMT of airway epithelial cells in CSexposed mice

To confirm that Lyn played a key role in regulation of EMT in CS-induced COPD, $\mathrm{Lyn}^{+/+}$transgenic mice model were constructed and lung injuries were checked. In control and $\mathrm{Lyn}^{+/+}$transgenic mice, no obvious lesions were 


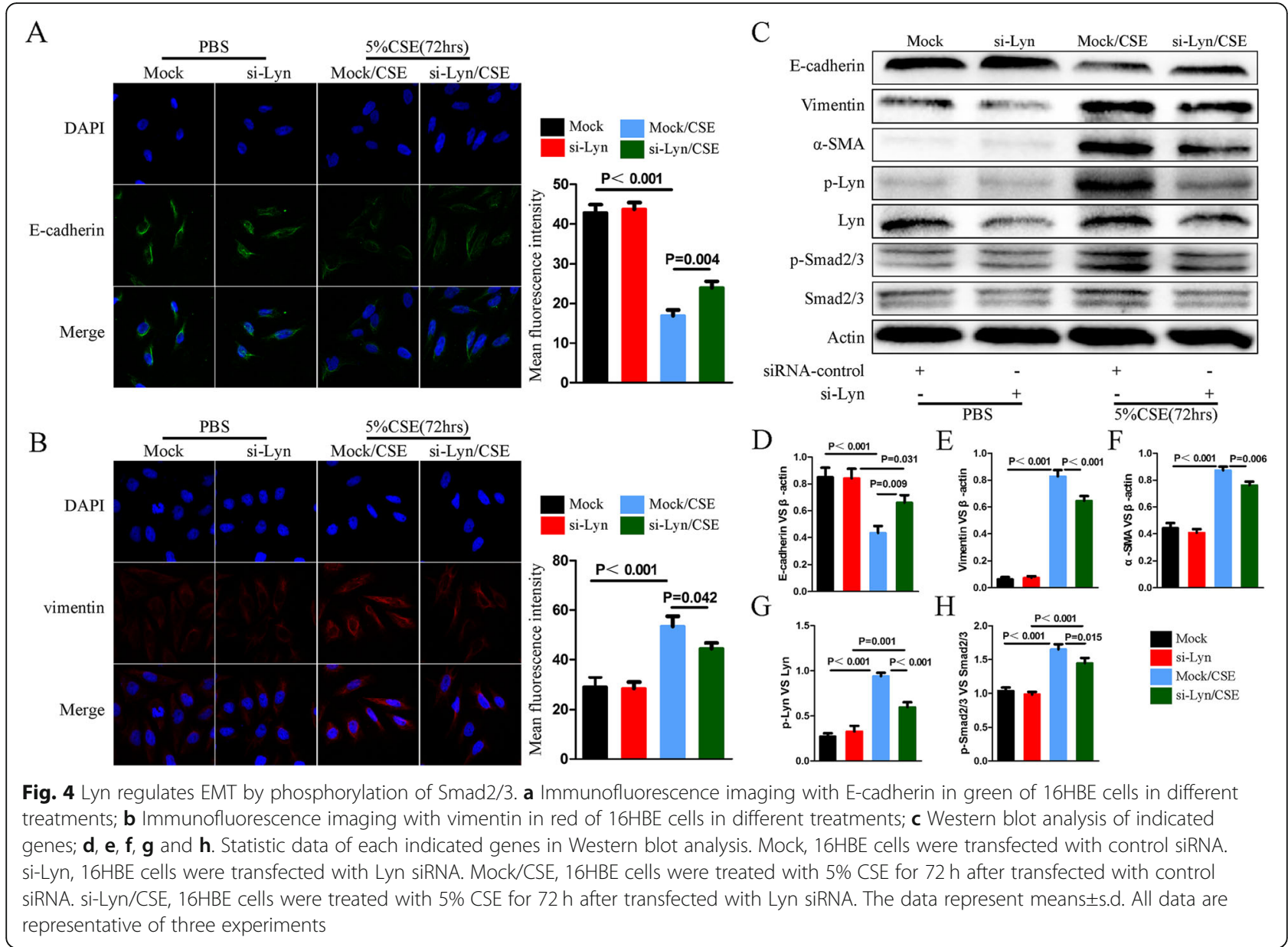

observed in the lung, and alveolar walls were intact (Fig. 5a, b). But in CS-exposed and $\mathrm{Lyn}^{+/+} / \mathrm{CS}$ groups, the lung had severe lesions (Fig. 5c, d). Additionally, especially in $\mathrm{Lyn}^{+/+} / \mathrm{CS}$ group, the alveolar walls were thin, the alveoli were expanded (Fig. 5d). In addition, the severity of regional emphysema was measured by mean linear intercept (MLI), $\mathrm{Lyn}^{+/+}$mice exposed to CS had more severe emphysema than any other groups (Fig. 5e). These results showed that typical pathological changes occurred after long-term exposure to CS in mice, especially in $\mathrm{Lyn}^{+/+}$ transgenic mice.

In the meantime, the expression levels of EMT makers in airway epithelial cells were assessed by using immunofluorescence. Compared with those in control mice, the fluorescence intensities of vimentin and $\alpha$ SMA in CS-exposed and $\mathrm{Lyn}^{+/+} / \mathrm{CS}$ mice were up-regulated, whereas the fluorescence intensities of E-cadherin were down-regulated in CS-exposed and $\mathrm{Lyn}^{+/+} / \mathrm{CS}$ mice compared with those in control mice (Fig. 6a). Furthermore, western blot was performed to detect the protein levels of EMT markers(Fig. 6b). The protein levels of vimentin and $\alpha$-SMA were increased in CS-exposed and $\mathrm{Lyn}^{+/+} / \mathrm{CS}$ mice compared with control mice, with the decreased protein levels of E-cadherin. It also showed that the phosphorylation levels of Lyn and Smad2/3 were increased in CS-exposed and $\mathrm{Lyn}^{+/+} / \mathrm{CS}$ mice compared with non-treated groups including control and $\mathrm{Lyn}^{+/+}$mice.

\section{Discussion}

COPD is in the spotlight for the high prevalence and morbidity, and the high burden of COPD associated with cigarette smoking has been increasing worldwide [29]. A study of systematic review and meta-analysis showed that the prevalence rate of COPD was obviously higher in smokers compared with that in non-smokers during 1990-2004 across 28 countries, and COPD affected up to $50 \%$ of all long-term smokers [30-32]. It is believed that cigarette smoking contributes to structural changes in the airways during COPD progression and initiates airway remodeling, a well-recognized pathological feature of COPD [4, 33]. Indeed, in our present study, pathological changes and obvious lesions were observed in the lung in CS-exposed mice compared with those in WT mice. Furthermore, in CS-induced groups, alveolar walls were thin, alveoli were expanded, more 

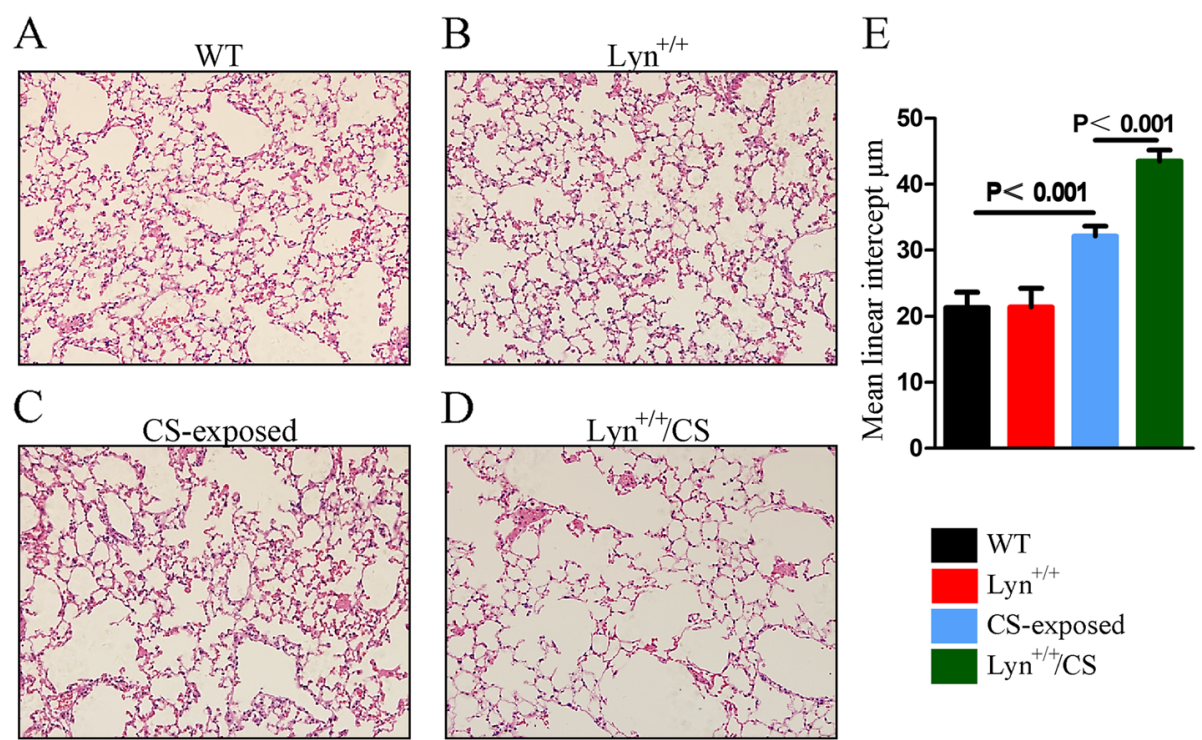

Fig. 5 Lyn enhances pathological changes of lung in CS-exposed mice.a Wild type mice; $\mathbf{b}$ Lyn over-expression transgenic mice; c CS-exposed mice model in WT background; $\mathbf{d}$ CS-exposed mice model in $\mathrm{Lyn}^{+/+}$background. e Regional emphysema severity was quantified in each sample using the mean linear intercept. WT, wild type mice; Lyn ${ }^{+/+}$, Lyn over-expression transgenic mice; CS-exposed, CS-exposed mice model in WT background; $\mathrm{Lyn}^{+/+} / \mathrm{CS}$, CS-exposed mice model in $\mathrm{Lyn}^{+/+}$background. All data are representative of three experiments

severe emphysema was observed. It confirmed that cigarette smoking is one of the main risk factors for COPD.

EMT is involved in changes that lead to loss of cellcell adhesion and cell polarity, with acquisition of migratory and invasive properties, which occurs during wound healing, tissue regeneration, organ fibrosis and tumor progression. Now EMT is thought to be involved in COPD pathology, which could contribute to small airway narrowing and airflow obstruction $[34,35]$. The gain of mesenchymal markers like vimentin and $\alpha$-SMA with a concomitant loss of E-cadherin are hallmarks of EMT. Recent studies showed that cigarette smoking induced EMT in alveolar type II cell line A549 and bronchial epithelial cell line BEAS2B [36, 37]. In our present study, in order to estimate the effect of CSE on EMT, 16HBE cells were treated with CSE at different time-points or concentrations. The result showed that CSE induced EMT in 16HBE cells in a time- and dose-dependent manner. Meanwhile, in vivo study also confirmed that EMT was able to be induced in CS-induced groups. Therefore, EMT might be a new target in anti-COPD drug discovery.

Src family kinases (SFKs) are the largest family of nonreceptor tyrosine kinases, which have a major role in cellular events of different biological systems, such as immune system and nervous system [38, 39]. Besides the fundamental roles in cell proliferation and survival, SFKs enhance cell migration and invasion across different cancers through EMT [40]. Lyn is one of the SFK members, functions as molecular switches that control immune receptors to direct homeostasis or inflammation. Studies have shown that there are correlations between increased expression of Lyn and mesenchymal phenotype in cancer cells [41, 42]. However, a correlation between expression of Lyn and EMT in CE-induced COPD has not been defined well. By analyzing GEO datasets, we found the expression level of Lyn was up-regulated, and the expression of EMT markers like vimentin and $\alpha$ SMA were increased as well. Although the expression level of E-cadherin was not changed in this GEO data, previous studies have revealed that its expression was decreased in COPD patients and we also validated it in the cell and animal models [6, 7]. Furthermore, the increasing range of vimentin and $\alpha$-SMA, and the decreasing range of E-cadherin were enhanced in $\mathrm{Lyn}^{+/+} / \mathrm{CS}$ mice and in $\mathrm{Lyn}^{+/+}$cells treated with CSE. In the meantime, after knockdown Lyn using siRNA, the expression levels of EMT markers were recovered in CSE treatment. According to these data, we believed that the expression of Lyn correlates with the expression of E-cadherin, vimentin and $\alpha$-SMA in CS-induced COPD.

Like other Src family members, Lyn has a kinase domain along with two protein interaction domains. Lyn regulates various cellular processes by phosphorylation of inhibitory receptors, enzymes, and adaptors through its kinase activity [43, 44]. In our study, we discovered not only the expression of Lyn was increased in CSexposed model, but also the phosphorylation of Lyn was up-regulated. However, no changes in phosphorylation of Lyn were observed in non-treatment group, neither in $\mathrm{Lyn}^{+/+}$mice nor in $\mathrm{Lyn}^{+/+}$cells. This leads us to 


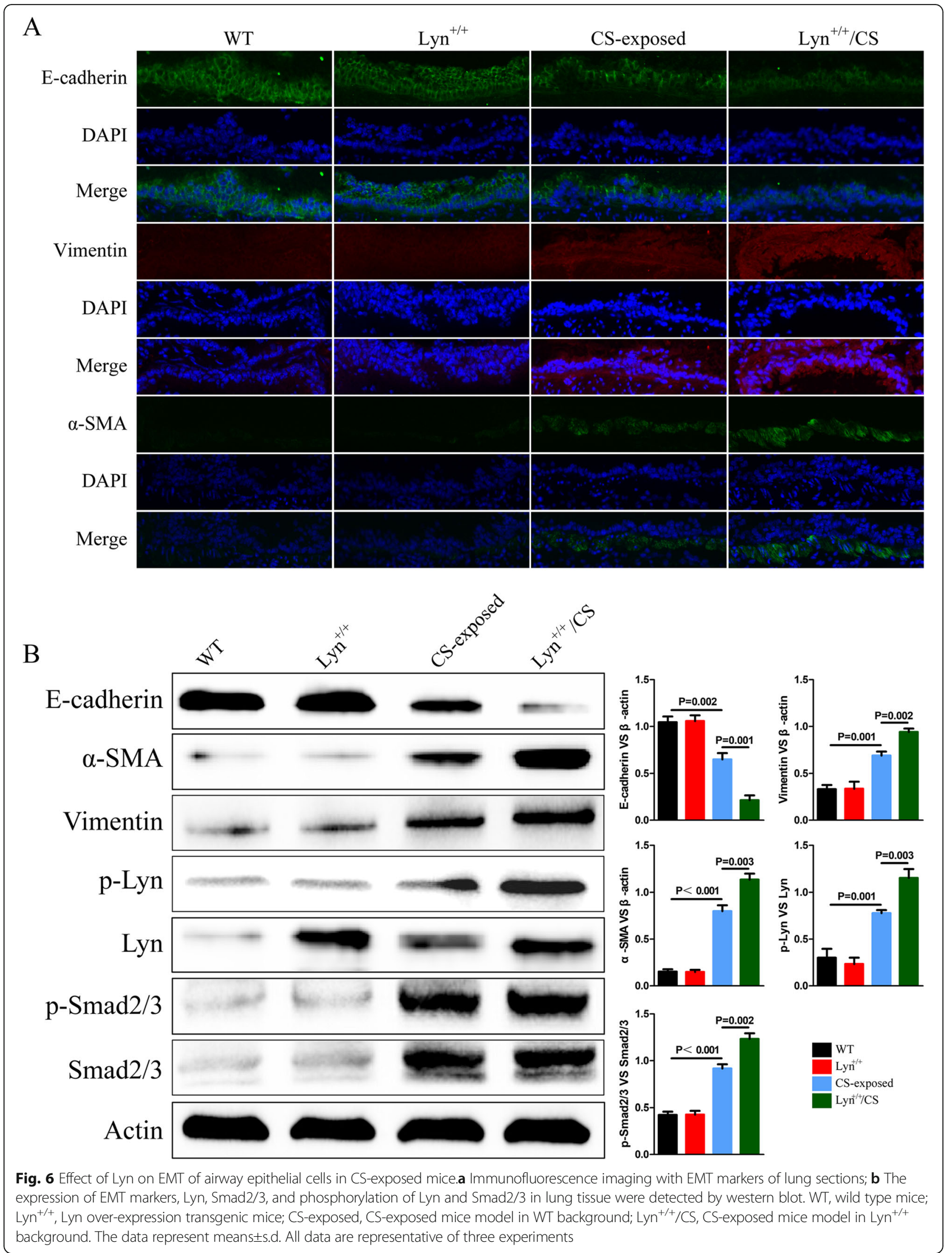




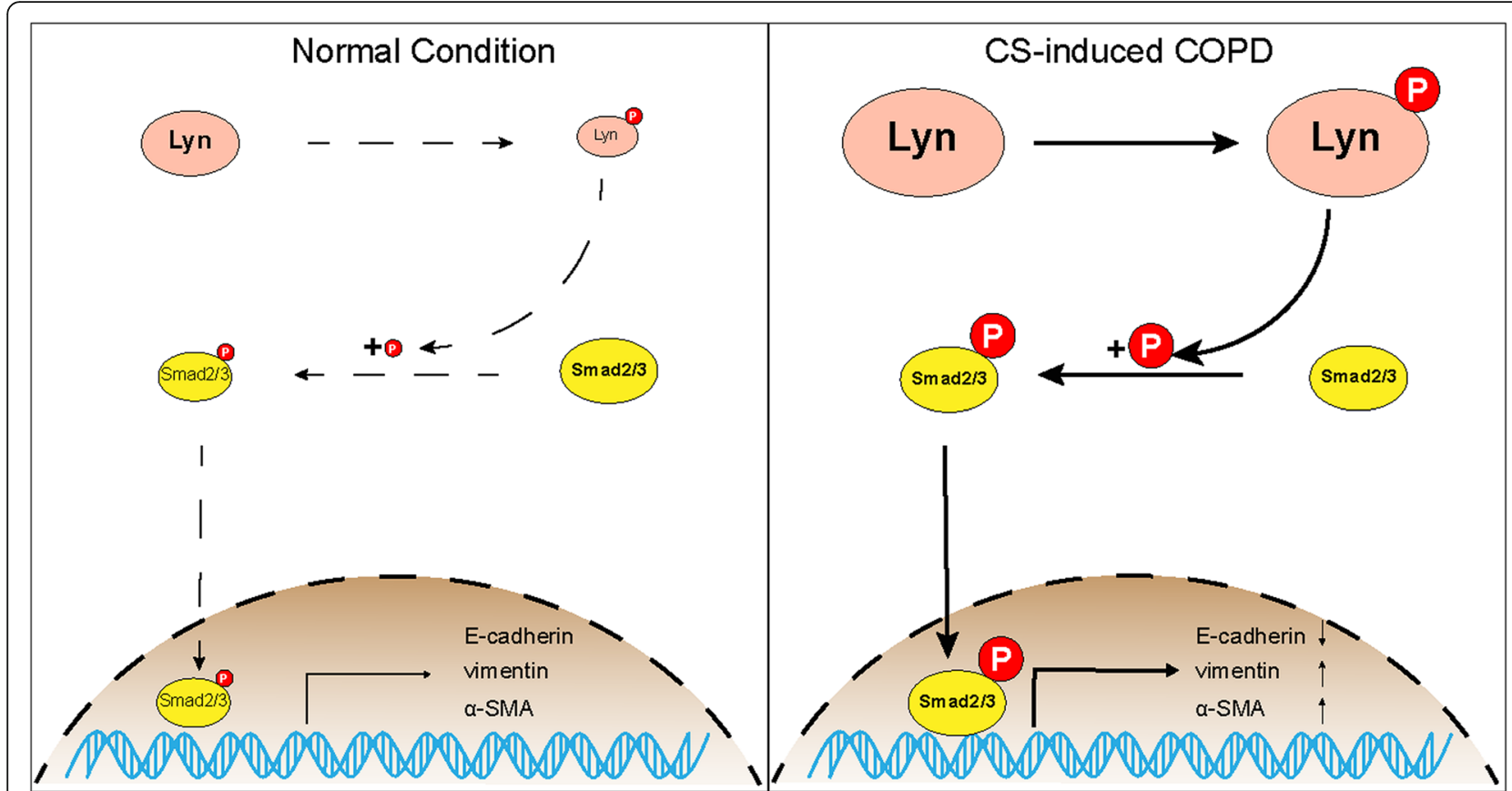

Fig. 7 Schematic of Lyn regulates EMT through Smad2/3 signaling in CS-exposed model. In CS-exposed model, not only the expression of Lyn, but also the phosphorylation of Lyn was increased. Then phosphorylation of Smad2/3 was up-regulated by activated Lyn through a direct or indirect way, and more phosphorylated Smad2/3 moved into nucleus. In nucleus, phosphorylated Smad2/3 functioned as major transcription factors to regulate the expression of EMT makers, promoted EMT in CS-exposed model

convince that the phosphorylation level of Lyn plays a key role in promoting EMT in CS-exposed model. Importantly, the phosphorylation of Smad2/3 was enhanced by activated Lyn in $\mathrm{Lyn}^{+/+} / \mathrm{CS}$ mice and in $\mathrm{Lyn}^{+/+}$cells treated with CSE, and the phosphorylation of Smad2/3 was decreased after Lyn knockdown in CSE treatment. On one hand, we speculated that Lyn was able to phosphorylate Smad2/3 directly through its kinase activity, and there might be protein-protein interactions between Lyn and Smad2/3. On the other hand, Lyn phosphorylated Smad2/3 in an indirect manner, in which Lyn functioned as a mediator to regulate the increasing phosphorylation of Smad2/3. But both of the hypotheses need to be further validated. In addition, transforming growth factor (TGF)- $\beta$, a multifunctional cytokine, has been implicated as a driver of COPD airway pathology $[45,46]$. It is widely known that TGF- $\beta$ activates the phosphorylation of Smad2/3 by binding to its receptors. Then the phosphorylated Smad2/3 enters into nucleus with Smad4, and regulates the expression levels of downstream genes. Therefore, there might be a potential relationship between TGF- $\beta$ and Lyn. The possible role of TGF- $\beta$ in regulation of Lyn or the function of Lyn in TGF- $\beta$ signaling pathway still needs to be investigated.

In summary, in CS-exposed model, not only the expression of Lyn, but also the phosphorylation of Lyn was increased. Then phosphorylation of $\mathrm{Smad} 2 / 3$ was upregulated by activated Lyn through a direct or indirect way, and more phosphorylated Smad2/3 moved into nucleus. In nucleus, phosphorylated Smad2/3 functioned as major transcription factors to regulate the expression of EMT makers, promoted EMT in CS-exposed model (Fig. 7). Current methods for treating COPD are longterm drug maintenance with inhaled corticosteroid and bronchodilators. In this study, we found that EMT in COPD is regulated by activated Lyn through phosphorylation of Smad2/3. Therefore, specific targeting of Lyn might be a potential therapeutic approach to treat COPD by inhibition of EMT.

\section{Conclusion}

By analyzing GEO data, we found that high expression of Lyn was associated with adherens junction. Lyn expression was up-regulated in COPD-smoker patients, which was validated by western blot. In addition, the gain of vimentin and $\alpha$-SMA with a concomitant loss of E-cadherin correlated with Lyn expression in both cell and mouse models. Importantly, we found that Lyn regulated EMT by phosphorylation of Smad2/3. As a kinase, Lyn is "druggable". Therefore, our research might provide a new method to treat COPD by targeting Lyn kinase specifically.

\section{Abbreviations}

COPD: Chronic obstructive pulmonary disease; CSE: Cigarette smoking extracts; EMT: Epithelial-mesenchymal transition; GEO: Gene expression 
omnibus; H\&E: Hematoxylin-eosin staining; LPS: Lipopolysaccharide; SFKs: Src family kinases; WT: Wild type

\section{Acknowledgements}

We thank Yi Zhang, Linqiao Tang, and Jinkui Pi from Research Core Facility of West China Hospital for technical assistance.

\section{Author contributions}

Designed the study: GPL and XH. Performed the experiments: XBL, XFY and JYW. Wrote the paper: GPL, YL and XH. Analyzed the data: XBL, XYW and $\mathrm{DHW}$. Bioinformatics and Computational analysis were performed by $\mathrm{XH}, \mathrm{YL}$ and GPL. All authors read and approved the final manucript.

\section{Funding}

This project was supported by Health Commission of Sichuan Province (18PJ012, 19ZD002), and Science and Technology Department of Sichuan Province (2018JY0380). Luzhou government and Southwest Medical University Cooperative Project (No.2016lzxnyd-z01). National Nature Science Foundation of China (NO. 81970026).

\section{Availability of data and materials}

The datasets generated during and/or analyzed during the current study are available from the corresponding author on reasonable request.

\section{Ethics approval and consent to participate}

All animal experiments were performed and approved in accordance with the guidelines of the Institutional Animal Care and Use Committee in Southwest Medical University. Human study was authorized by the Ethics Committee of Southwest Medical University, and obtained written informed consents from all the participants.

\section{Consent for publication}

Not applicable.

\section{Competing interests}

The authors declare that they have no competing interests.

\section{Author details}

1 Inflammation \& Allergic Diseases Research Unit, Affiliated Hospital of Southwest Medical University, Luzhou 646000, China. ${ }^{2}$ First Department of Respiratory Disease, Affiliated Hospital of Southwest Medical University, Luzhou 646000, China. ${ }^{3}$ Laboratory of Allergy and Inflammation of Allergy Department, Chengdu Institute of Respiratory Health, the Third People's Hospital of Chengdu, Affiliated Hospital of Southwest Jiaotong University, Chengdu 610031, China. ${ }^{4}$ Department of Respiratory Disease, the Third People's Hospital of Chengdu, Affiliated Hospital of Southwest Jiaotong University, Chengdu 610031, China. ${ }^{5}$ Department of Thoracic Surgery, Zhongshan Hospital, Affiliated Hospital of Fudan University, Shanghai 200032, China.

\section{Received: 25 April 2019 Accepted: 15 August 2019}

Published online: 02 September 2019

\section{References}

1. WH O. World health statistics. World Health Organization; 2010. p. 2010.

2. Eisner MD, Anthonisen N, Coultas D, Kuenzli N, Perez-Padilla R, Postma D, et al. An official American Thoracic Society public policy statement: novel risk factors and the global burden of chronic obstructive pulmonary disease. Am J Respir Crit Care Med. 2010;182(5):693-718.

3. Zhong N, Wang C, Yao W, Chen P, Kang J, Huang S, et al. Prevalence of chronic obstructive pulmonary disease in China : a large, population-based survey. Am J Respir Crit Care Med. 2007;176(8):753.

4. Hogg JC, Chu F, Utokaparch S, Woods R, Elliott WM, Buzatu L, et al. The nature of small-airway obstruction in chronic obstructive pulmonary disease. N Engl J Med. 2004;350(26):2645.

5. Hirota N, Martin JG. Mechanisms of airway remodeling. Chest. 2013;144(3): 1026-32.

6. Milara J, Peiró T, Serrano A, Cortijo J. Epithelial to mesenchymal transition is increased in patients with COPD and induced by cigarette smoke. Thorax. 2013;68(5):410-20
7. Gohy ST, Hupin C, Fregimilicka C, Detry BR, Bouzin C, Gaide CH. Imprinting of the COPD airway epithelium for dedifferentiation and mesenchymal transition. Eur Respir J. 2015;45(5):1258-72.

8. Domokos B, Nikica M, Mahida RY, Oliver E, Thickett DR. Epithelialmesenchymal transition in lung development and disease: does it exist and is it important? Thorax. 2014;69(8):760-5.

9. Raghu K. Weinberg RA. Journal of Clinical Investigation: The basics of epithelial-mesenchymal transition; 2015.

10. Nieto MA, Huang RYJ, Jackson R, Thiery JP. EMT: 2016. Cell. 2016;166(1):2145.

11. Samy L, Jian X, Rik D. Molecular mechanisms of epithelial-mesenchymal transition. Nat Rev Mol Cell Biol. 2014;15(3):178-96.

12. Mahmood MQ, Reid D, Ward C, Muller HK, Knight DA, Sohal SS, et al. Transforming growth factor (TGF) $\beta$ and Smad signalling pathways: a likely key to EMT-associated COPD pathogenesis. Respirology. 2017;22(1):133.

13. Bertero A, Brown S, Madrigal P, Osnato A, Ortmann D, Yiangou L, et al. The SMAD2/3 interactome reveals that TGF $\beta$ controls m6A mRNA methylation in pluripotency. Nature. 2018;555(7695):256-9.

14. Yeh HW, Hsu EC, Lee SS, Lang YD, Lin YC, Chang CY, et al. PSPC1 mediates TGF- $\beta 1$ autocrine signalling and Smad2/3 target switching to promote EMT, stemness and metastasis. Nat Cell Biol. 2018;20(4):479-91.

15. Zhou Q, Zheng X, Chen L, Xu B, Yang X, Jiang J, et al. Smad2/3/4 pathway contributes to TGF- $\beta$-induced MiRNA-181b expression to promote gastric Cancer metastasis by targeting Timp3. Cell Physiol Biochem. 2016;39(2):45366.

16. Hui F, He Y, Qi L, Lu C, Yi L, Chen L, et al. CPLA2a activates PI3KAKT and inhibits Smad2/3 during epithelial\&ndash;mesenchymal transition of hepatocellular carcinoma cells. Cancer Lett. 2017;403:S0304383517304044.

17. Tian F, Wt DCBS, Yoo S, Felici A, Tang B, Piek E, et al. Reduction in Smad2/3 signaling enhances tumorigenesis but suppresses metastasis of breast cancer cell lines. Cancer Res. 2003;63(23):8284-92.

18. Grishin AV, Azhipa O, ., Semenov I, ., Corey SJ. Interaction between growth arrest-DNA damage protein 34 and Src kinase Lyn negatively regulates genotoxic apoptosis. Proc Natl Acad Sci U S A 2001;98(18):10172-10177.

19. Ban T, Sato GR, Nishiyama A, Akiyama A, Takasuna M, Umehara M, et al. Lyn kinase suppresses the transcriptional activity of IRF5 in the TLR-MyD88 pathway to restrain the development of autoimmunity. Immunity. 2016; 45(2):319-32.

20. Li G, Fox J, Liu Z, Liu J, Gao GF, Jin Y, et al. Lyn mitigates mouse airway remodeling by downregulating the TGF- $\beta 3$ isoform in house dust mite models. J Immunol(Baltimore, Md : 1950). 2013;191(11):5359-70.

21. Marcello G. Src signaling in cancer invasion. J Cell Physiol. 2010;223(1): $14-26$.

22. Wang $X$, Li Y, Luo D, Wang X, Zhang Y, Liu Z, et al. Lyn regulates mucus secretion and MUC5AC via the STAT6 signaling pathway during allergic airway inflammation. Sci Rep. 2017;7:42675.

23. Laurila E, Mootha VK, Lindgren CM, Eriksson KF, Subramanian A, Sihag S, et al. PGC-1a-responsive genes involved in oxidative phosphorylation are coordinately downregulated in human diabetes. Nat Genet. 2003:34(3):267-73.

24. Aravind Subramanian PT, Vamsi K, Mootha, Mukherjee S, Ebert BL, Gillette MA, Paulovich A, Pomeroy SL, Golub TR, Lander ES, Mesirov JP. Gene set enrichment analysis: a knowledge-based approach for interpreting genomewide expression profiles. Proc Natl Acad Sci U S A. 2005:102(43):15545-50.

25. Kyoung-Hee L, Chang-Hoon L, Jiyeong J, An-Hee J, Chul-Gyu Y. Neutrophil elastase differentially regulates interleukin 8 (IL-8) and vascular endothelial growth factor (VEGF) production by cigarette smoke extract. J Biol Chem. 2015;290(47):28438

26. Zhi-Hui H, Ping C, Yan C, Sheng-Dong H, Ji-Ru Y, Hong-Liang Z, et al. Comparison between cigarette smoke-induced emphysema and cigarette smoke extract-induced emphysema. Tob Induc Dis. 2015;13(1):1-8.

27. Cortijo J, Mata M, Milara J, Donet E, Gavaldà A, Miralpeix M, et al. Aclidinium inhibits cholinergic and tobacco smoke-induced MUC5AC in human airways. Eur Respir J. 2011;37(2):244-54

28. Thien A, Prentzell MT, Holzwarth B, Kläsener K, Kuper I, Boehlke C, et al. TSC1 activates TGF- $\beta$-Smad2/3 signaling in growth arrest and epithelial-tomesenchymal transition. Dev Cell. 2015:32(5):617-30.

29. López-Campos JL, Tan W, Soriano JB. Global burden of COPD. Respirology 2016;21(1):14-23.

30. Halbert RJ, Natoli JL, Gano A, ., Badamgarav E, ., Buist AS, Mannino DM. Global burden of COPD: systematic review and meta-analysis. Int Braz J Urol Off J Braz Soc Urol 2006;39(6):768. 
31. Lundbäck B, Lindberg A, Lindström $M$, Rönmark $E$, Jonsson $A C$, Jönsson $E$, et al. Not 15 but $50 \%$ of smokers develop COPD? - report from the obstructive lung disease in northern Sweden studies. Respir Med. 2003;97(2):115-22.

32. Laniadolaborín R. Smoking and chronic obstructive pulmonary disease (COPD). Parallel epidemics of the 21st century. Int J Environ Res Public Health. 2009;6(1):209-24.

33. Sara K, Umme K, Karine R, Dave S, Jørgen V, Thornton DJ. MUC5B is the major mucin in the gel phase of sputum in chronic obstructive pulmonary disease. Am J Respir Crit Care Med. 2008;178(10):1033-9.

34. Javier M, Rafael N, Gustavo J, Teresa P, Adela S, Mercedes R, et al. Sphingosine-1-phosphate is increased in patients with idiopathic pulmonary fibrosis and mediates epithelial to mesenchymal transition. Thorax. 2012; 67(2):147-56.

35. Kaosia N, Sukhwinder Singh S, Gregory P, Rahul P, Eugene HW. Epithelialmesenchymal transition as a fundamental underlying pathogenic process in COPD airways: fibrosis, remodeling and cancer. Expert Rev Respir Med. 2014; 8(5):547-59.

36. Liu Y, Gao W, Zhang D. Effects of cigarette smoke extract on A549 cells and human lung fibroblasts treated with transforming growth factor- $\beta 1$ in a coculture system. Clin Exp Med. 2010;10(3):159-67.

37. Veljkovic E, Jiricny J, Menigatti M, Rehrauer H, Han W. Chronic exposure to cigarette smoke condensate in vitro induces epithelial to mesenchymal transition-like changes in human bronchial epithelial cells, BEAS-2B. Toxicol Vitro Int J Published in Assoc Bibra. 2011:25(2):446-53.

38. Lowell CA. Src-family kinases: rheostats of immune cell signaling. Mol Immunol. 2004:41(6):631-43.

39. Maness PF, Beggs HE, Klinz SG, Morse WR. Selective neural cell adhesion molecule signaling by Src family tyrosine kinases and tyrosine phosphatases. Perspect Dev Neurobiol. 1996;4(2-3):169-81.

40. Huang RY. Epithelial-mesenchymal transitions in development and disease. Cell. 2009;139(5):871-90.

41. Guan H, Zhou Z, Gallick GE, Jia SF, Morales J, Sood AK, et al. Targeting Lyn inhibits tumor growth and metastasis in Ewing's sarcoma. Mol Cancer Ther. 2008; $7(7): 1807$

42. Yoon-La C, Melanie B, Jeong KM, Young Kee S, Seok Jin N, Jung-Hyun Y, et al. LYN is a mediator of epithelial-mesenchymal transition and a target of dasatinib in breast cancer. Cancer Res. 2010:70(70):2296-306.

43. Ji W, Feng M, Henry L, Ling K, William B, Zhenghong $\mathrm{P}$, et al. Lyn regulates BCR-ABL and Gab2 tyrosine phosphorylation and c-Cbl protein stability in imatinib-resistant chronic myelogenous leukemia cells. Blood. 2008;111(7): 3821-9.

44. Wu R, Zeng J, Yuan J, Deng X, Huang Y, Chen L, et al. MicroRNA-210 overexpression promotes psoriasis-like inflammation by inducing Th1 and Th17 cell differentiation. J Clin Invest. 2018:128(6):2551-68.

45. Minagawa S, Lou J, Seed Rl, Cormier LA, Wu RSS, Cheng Y, et al. Selective Targeting of TGF- Activation to Treat Fibroinflammatory Airway Disease. Sci Transl Med. 2014;6(241):241-ra79.

46. Hogg J. Pathophysiology of airflow limitation in chronic obstructive pulmonary disease. Lancet (London, England). 2004;364(9435):709-21.

\section{Publisher's Note}

Springer Nature remains neutral with regard to jurisdictional claims in published maps and institutional affiliations.

Ready to submit your research? Choose BMC and benefit from:

- fast, convenient online submission

- thorough peer review by experienced researchers in your field

- rapid publication on acceptance

- support for research data, including large and complex data types

- gold Open Access which fosters wider collaboration and increased citations

- maximum visibility for your research: over $100 \mathrm{M}$ website views per year

At $\mathrm{BMC}$, research is always in progress.

Learn more biomedcentral.com/submissions 